\title{
Terahertz Spectroscopy and Imaging Detection of Defects in Civil Aircraft Composites
}

\author{
Mian Zhong, ${ }^{1}$ Bingwei Liu, ${ }^{1}$ Chen Li $\left(\mathbb{D},{ }^{2}\right.$ Zhiqi Wang, ${ }^{2}$ Dongshan Wei $\mathbb{D},{ }^{3}$ Bin Zhou $\left(\mathbb{D},{ }^{4}\right.$ \\ Xin Dai, ${ }^{1}$ and Yajun $\mathrm{Xu}\left(\mathbb{D}^{1}\right.$ \\ ${ }^{1}$ Aviation Engineering Institute, Civil Aviation Flight University of China, Guanghan 618307, China \\ ${ }^{2}$ Shenzhen Institute of Terahertz Technology and Innovation, Shenzhen 518110, China \\ ${ }^{3}$ School of Electrical Engineering and Intelligentization, Dongguan University of Technology, Dongguan 523808, \\ Guangdong, China \\ ${ }^{4}$ NDT \& Calibration Centre, Beijing Aircraft Maintenance and Engineering Corporation Chengdu Branch, \\ Chengdu 610202, China
}

Correspondence should be addressed to Chen Li; lichen@huaxunchina.cn, Dongshan Wei; dswei@dgut.edu.cn, and Yajun Xu; genius98@126.com

Received 18 September 2019; Revised 11 December 2019; Accepted 4 January 2020; Published 20 February 2020

Academic Editor: Rizwan Hasan Khan

Copyright (c) 2020 Mian Zhong et al. This is an open access article distributed under the Creative Commons Attribution License, which permits unrestricted use, distribution, and reproduction in any medium, provided the original work is properly cited.

Composite materials have increasingly become a high proportion of the structural weight of aircraft due to their excellent performances. Different types of damages may occur in the aircraft service period, which will bring potential safety risks to aircrafts. To investigate the defect damage detection and its spectral characteristics and imaging of carbon-fiber-reinforced polymer composite laminates, defects from the low-velocity impact damage in composites were measured by the THz timedomain reflection imaging system. Results show that there exists obvious $\mathrm{THz}$ spectral differences between the impact damaged defects and nondefect. The effective detection frequency band for the low-speed impact damaged defect is $0.12-2.0 \mathrm{THz}$. In the time domain, there are attenuations and delays in the spectra of defects relative to those of nondefects. In the frequency domain, with the increase of frequency, the power spectral density of the defect first increases and then decreases, and the absorption coefficient increases slowly. In general, the imaging results in time-domain imaging are better than those from the frequencydomain imaging, which not only is suitable for the qualitative detection of defects but also has great potential and application prospects in quantitative detection. This work shows an important guide for the application of $\mathrm{THz}$ technology to detect the composite material defects in civil aircraft.

\section{Introduction}

Composite materials have been widely used in the aviation field because of their high specific strength, stiffness, and good fatigue and corrosion resistances, and their proportion in the structural weight of aircraft has significantly increased $[1,2]$. Owing to the particularities of composite materials, different types of damage may occur in their production and service processes, such as pores, delamination, surface scratches and cracks, debonding, and degumming [3]. Compared with metals, the damage and failure modes of composite materials are more complicated [4]. The composite material components of in-service aircraft are vulnerable to low-speed impact damages caused by bird strikes, hail shocks, and impact of maintenance tools. The existence of these types of damage is an important factor leading to potential safety hazards in aircraft [5]. In addition, these types of damage are difficult to observe on the surface. With the help of other detection equipment, it is of great significance to use accurate and efficient nondestructive detection technologies to detect the low-speed impact damage of composite materials to ensure the safe operation of aircrafts [6,7].

Recently, many nondestructive testing technologies for civil aircraft exist, including laser ultrasonic methods $[8,9]$, 
infrared thermal imaging method [10-12], and electronic speckle pattern interferometry (ESPI) [13, 14]. However, these methods have their own disadvantages. For example, in ultrasonic detection, a coupling agent is needed, the signal-to-noise ratio of the received signal is poor due to the ultrasonic attenuation, and the corresponding detection probes must to be matched for different defects. The imaging detection resolution of an infrared thermal imager is severely limited by the performance of the detector itself, the cost of high-performance detection equipment is extremely high, and the quantitative analysis of defects often exhibits errors caused by the influence of thermal diffusion. The laser speckle technique has the problem of poor sensitivity to defects in materials and the difficulty in quantification.

A terahertz wave is an electromagnetic wave with frequency between 0.1 and $10 \mathrm{THz}$. With the development and application of terahertz sources and advanced terahertz testing equipment, the potential of terahertz technology in fabricating nonconductive material detection has been exploited, and it also has unique advantages in nondestructive testing of composites $[15,16]$. In recent years, researchers have carried out some studies in the field of composite material detection using terahertz technology. Hsu et al. used terahertz wave to nondestructively detect defects in glass-fiber-reinforced plastic laminates, and their results show that terahertz pulses can detect microcracks in such materials [17]. Jördens et al. investigated the fiber orientation and fiber content of glass-fiber-reinforced composites by terahertz time-domain spectroscopy, measured and analyzed their refractive properties, and verified the feasibility of terahertz time-domain spectroscopy and imaging technology for nondestructive testing of such composites [18]. Abina et al. studied the phase transformation process of microencapsulated phase-change materials by analyzing the terahertz spectra of polymer-foamreinforced plastics, and the defects inside the foam structure were clearly detected by terahertz amplitude imaging [19]. Xing et al. reported that the $0.05-0.6 \mathrm{THz}$ band is the effective detection frequency band of polymethacrylimide (PMI) foam composites by analyzing the terahertz spectral characteristics of two kinds of PMI foam composites [20]. Wang et al. studied the spectral characteristics and regularity of three kinds of aviation glass-fiber composites and their matrix resins in the range of $0.2-1.0 \mathrm{THz}$ band and provided a guide for the application of terahertz nondestructive testing in aviation composites [21]. However, to our knowledge, there is no report of the detection and analysis of low-velocity impact damage defects of carbon-fiber-reinforced polymer (CFRP) composites by terahertz spectroscopy and imaging technology.

In this work, terahertz time-domain spectroscopy and imaging technology were used to nondestructively detect low-velocity impact damage defects in CFRP composite laminates. The terahertz spectra of defects were extracted, and their characteristics were systematically analyzed. The time- and frequency-domain imaging displays of defects were obtained, and the detection of these defects was discriminated and analyzed.

\section{Experimental Details}

2.1. Experimental Specimen. The specimen studied was T300/Cycom 970 composite laminates with a laying mode of $[0 / 45 / 90 /-45]_{12}$ and dimensions of $210 \mathrm{~mm} \times 70 \mathrm{~mm} \times 2.6 \mathrm{~mm}$, which was provided by Xi'an Aircraft Industry (Group) Company. The fiber was a cross-braided fabric, and the thickness of a single prepreg was $0.216 \mathrm{~mm}$. The material was adopted for Boeing 747-8 inner flaps and passenger aircraft girders. According to the ASTMD7136/D7136M-07 specification, the impact test was carried out with a drop hammer low-speed impact test device. The impact hammer was a steel hemisphere with a diameter of $12.7 \mathrm{~mm}$ and a mass of $1 \mathrm{~kg}$. The impact energy of the specimen was $3.67 \mathrm{~J}$, which simulates the free-fall impact phenomena occurring during actual maintenance processes due to impacts on the aircraft composite structure knives, wrenches, and other maintenance tools.

\subsection{Experimental Apparatus. A CCT-1800 series terahertz} time-domain spectroscopy (THz-TDS) imaging system (China Communication Technology (CCT) Co., China) was used, in addition to a terahertz time-domain spectral analyzer, reflection imaging module and data-acquisition system, and analysis software. A schematic of the THz-TDS imaging system in reflection mode is shown in Figure 1. A $780 \mathrm{~nm}$ fiber femtosecond laser (Menlo Systems, Germany) was used as excitation source in the system. Via the beam splitting mirror, the system was divided into pump and probe beams, which were transformed to terahertz waves with a stable excitation and reception spectrum ranging from 0.06 to $4 \mathrm{THz}$, respectively. The optical delay was $54 \mathrm{ps,}$ and the spectral resolution and dynamic range were up to $20 \mathrm{GHz}$ and $80 \mathrm{~dB}$, respectively. The THz-TDS reflection imaging mode was used to scan the specimens on a twodimensional platform. The sampling rate of waveforms was $15 \mathrm{~Hz}$, the minimum scanning step was $0.08 \mathrm{~mm}$, and the maximum scanning range was $50 \mathrm{~mm} \times 50 \mathrm{~mm}$.

2.3. Experimental Process. During the experiment, the ambient temperature and humidity were controlled at $23 \pm 1{ }^{\circ} \mathrm{C}$ and below $40 \%$, respectively. Nitrogen was continuously injected into the reflecting module to eliminate the interference of water vapor in the environment during tests. The incident $\mathrm{THz}$ wave emitted by the transmitter entered the test piece, which was placed at the sample table, i.e., the focal plane of the imaging system, returned with testing information, and was finally received by the receiver, as shown in Figure 1. The gold mirror was tested as a reference signal after nitrogen purging. After two-dimensional scanning, the $\mathrm{THz}$ time-domain pulse waveform and three-dimensional matrix data corresponding to each scanning point were obtained. Corresponding frequency-domain data were obtained by Fourier transform of the time-domain waveforms. After data analysis and processing in the time and frequency domains, maximum peak imaging, minimum peak imaging, maximum time-of-flight imaging, minimum time-of-flight imaging, peak-of-peak imaging, and spectral amplitude 


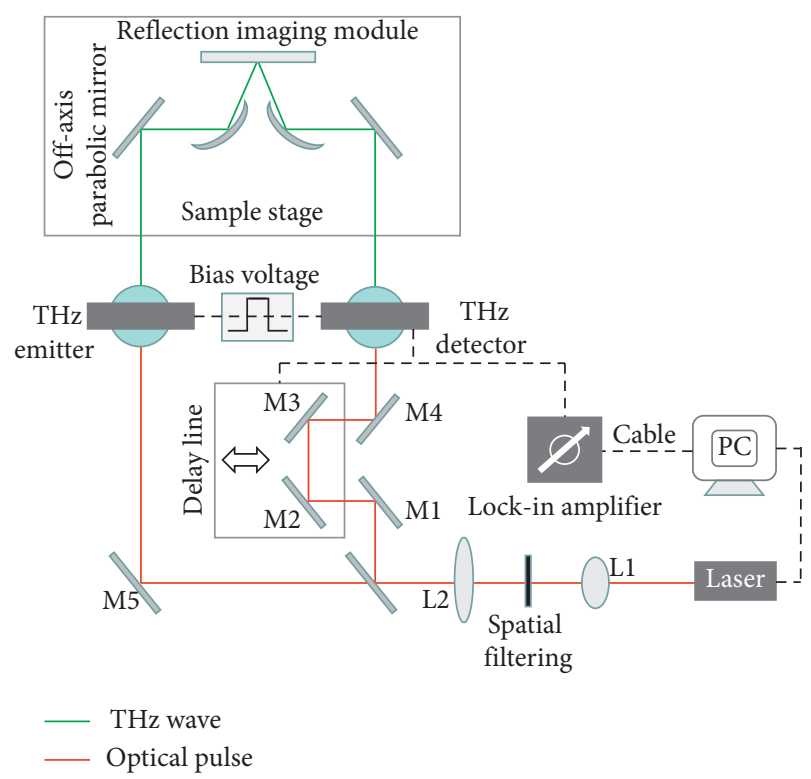

FIGURE 1: Schematic of the THz-TDS imaging system in reflection mode.

difference between defect and nondefect tests were performed according to the data characteristics of the terahertz spectra. In the frequency domain, the characteristic frequencies of power spectral density and absorption coefficient were analyzed and used for imaging, so as to realize nondestructive testing of specimens [22].

\section{Results and Discussion}

3.1. Time-Domain Waveform and Imaging. Figure 2 shows the terahertz time-domain signal waveforms of the defect and nondefect specimens. It can be seen from Figure 2(a) that the sample signal exhibits an amplitude attenuation and a time delay relative to the reference signal. In addition, the amplitude signal intensity at the defect site also has greater attenuation and time delay than those at the nondefect sites. The maximum amplitude of the defect site is approximately $32 \%$ of the nondefect sites, and the time delay is 1.19 ps. Because of the different reflection, transmission, and loss of the signal in the discontinuous interface composed of air or resin between composite layers, the amplitudes of the reflected signal that was detected are therefore different. The time delay in the reflection signals of the defective and nondefective parts is due to the different refractive indexes of air and resin. Figure 2(b) shows the difference in signal amplitude between the defect and nondefect sites in the time domain. On the time axis, the difference between the defect and nondefect sites at different times is quite different, and the imaging can be performed according to local eigenvalues.

Figure 3 shows the time-domain signal imaging results of the defect in the tested specimens. Figures 3(a)-3(e) present the imaging results based on the maximum peak, minimum peak, maximum flight time, minimum flight time, and peak value in the time domain, respectively. It is clear that these imaging results are obviously weaker than those obtained by choosing the characteristic time of the difference between the amplitudes of the defect and nondefect sites. As seen from Figures $3(f)-3(j)$, the former imaging only shows a small number of severely damaged structural areas, but does not fully show the impact damage defects in the specimen. Additionally, it has the shortcomings of low resolution and blurred edges. The result of amplitude difference imaging is due to the amplitude difference between $t=6.85 \mathrm{ps}$ and $t=11.89$ ps being small, the contrast between the defect and nondefect in corresponding images being weak, and the edge being blurred, which makes it difficult to completely distinguish the impact damage defect. However, the image contrast between the defect and the nondefect is obvious, and the edge is clear at $t=8.58,9.52$, and $10.68 \mathrm{ps}$, where the defect expands from the impact center to an approximately circular area. It is helpful to distinguish the impact damage in the specimen completely, so the defect can be detected quantitatively.

Because the amplitude difference was the largest when $t=9.52 \mathrm{ps}$, the image was used to segment and extract the defect, as shown in Figures 4(a) and 4(b), and the defect area was $210.125 \mathrm{~mm}^{2}$. We have used thermal imaging technology to detect the defect and obtained that the defect area is $202.742 \mathrm{~mm}^{2}$, which is shown in Figures 4(c) and $4(\mathrm{~d})$, respectively. It is can be found that the resolution of terahertz imaging is obviously higher than that of infrared imaging, and the edge is not fuzzy, which is beneficial to the extraction of defects. By comparing the results of the two technologies, the relative error is $3.6 \%$, which is satisfied with the damage detection requirements of CFRP composite in in-service aircraft. It is shown that terahertz time-domain spectroscopy and imaging technology has a higher accuracy in detecting such defects.

\subsection{Power Spectral Density Waveform and Imaging.} Figure 5 shows the power spectral density waveform and its amplitude difference between the defect and the nondefect sites in the specimen. It is clearly seen from Figure 5(a) that the general trend of reflection signals at the defect and nondefect in the frequency domain is to first increase and then decrease with increasing frequency. It is reported that the absorption and dissipation of polymer resin in terahertz band are low, mainly due to dipole polarization and relaxation dissipation [21]. The frequency-domain signal at the defect reaches its peak at $0.31 \mathrm{THz}$, and the frequency-domain signal at the nondefect reaches its peak at $0.39 \mathrm{THz}$, and the amplitude at the defect is relative to that at the nondefect. The attenuation of the defect contributes to the different absorption spectra of the interlayer resin and air in the terahertz frequency range, as well as to the light dispersion and absorption of the specimen structure itself and the defect. When the frequency is less than $0.12 \mathrm{THz}$, the frequency-domain signals are basically the same, and the two signals attenuate seriously after becoming larger than 2.0 THz, which contains a significant amount of interference noise. Therefore, the effective detection band for this defect is $0.12-2.0 \mathrm{THz}$. It can be seen in Figure 5(b) that the 


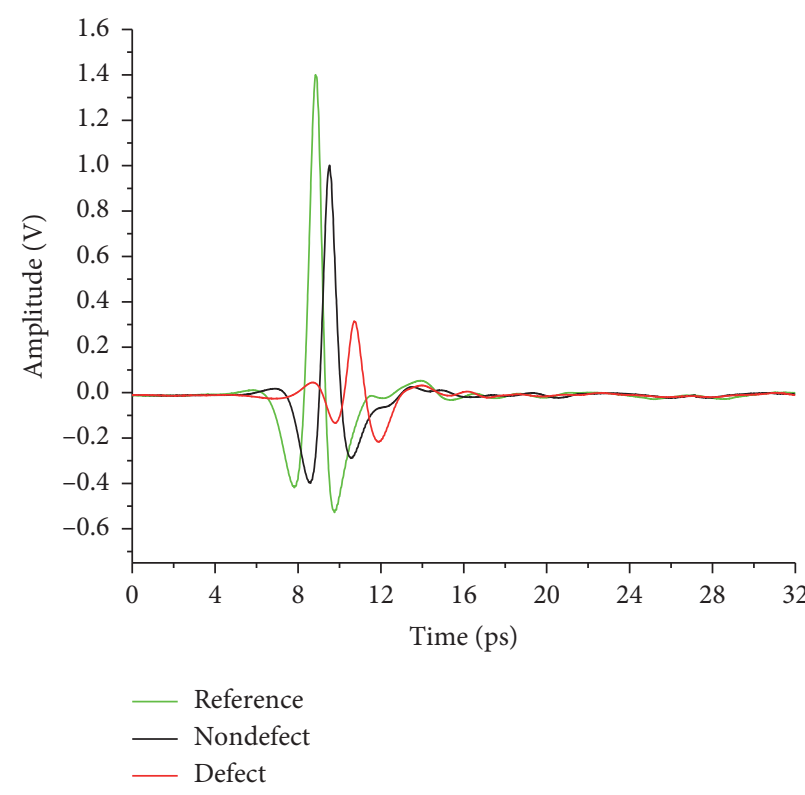

(a)

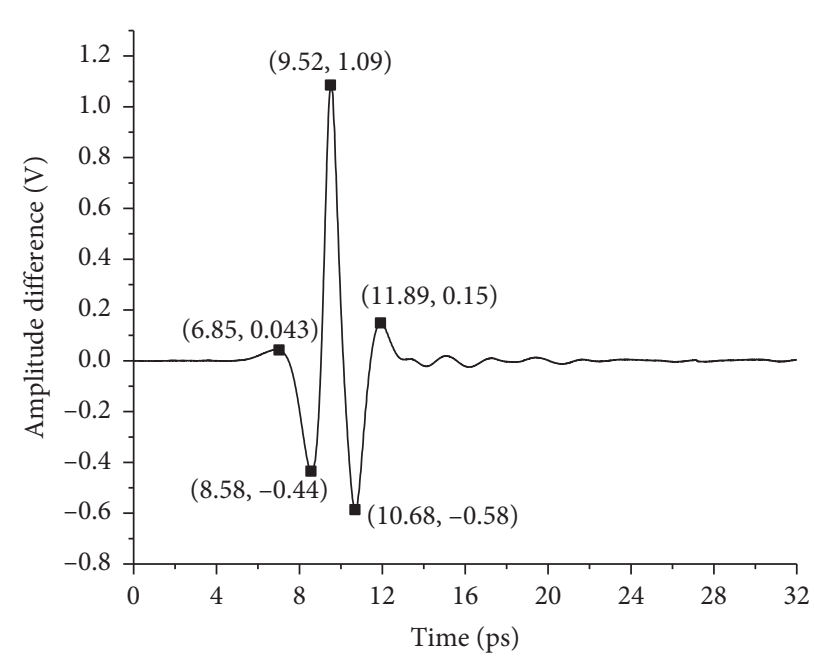

(b)

Figure 2: Terahertz waveforms of the experimental specimen: (a) time-domain waveform; (b) time-domain signal amplitude difference between the defect and nondefect sites.

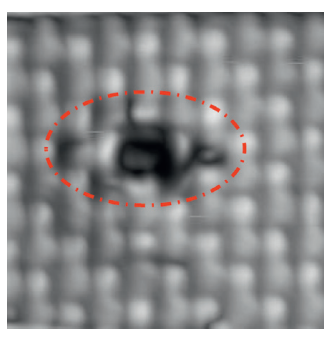

(a)

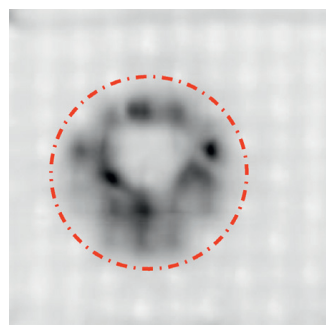

(f)

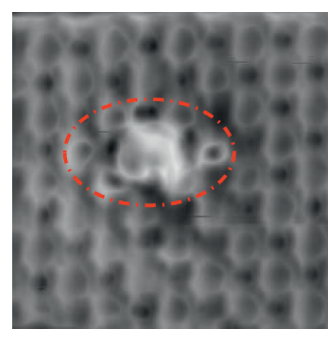

(b)

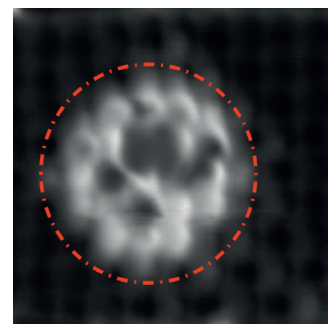

(g)

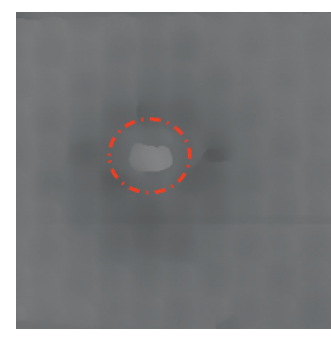

(c)

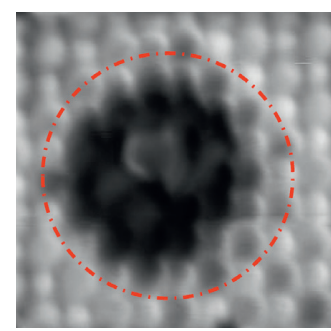

(h)

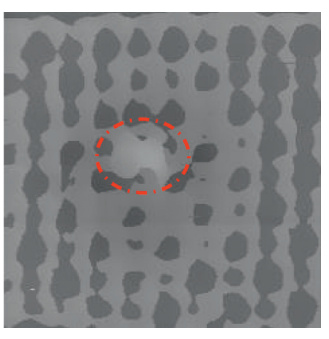

(d)

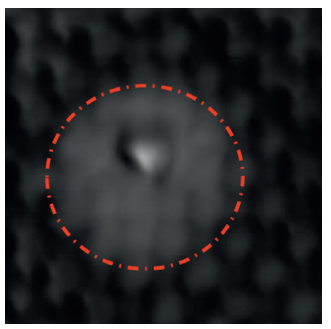

(i)

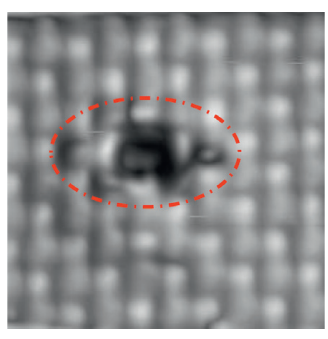

(e)

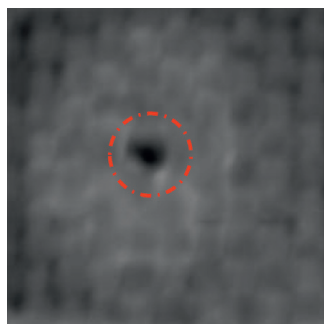

(j)

FIgURE 3: Time-domain signal imaging at defect of specimen: (a) time-domain maximum peak; (b) time-domain minimum peak; (c) maximum flight time; (d) minimum flight time; (e) peak-to-peak; (f) $t=6.85 \mathrm{ps}$; (g) $t=8.58 \mathrm{ps}$; (h) $t=9.52 \mathrm{ps}$; (i) $t=10.68 \mathrm{ps}$; (j) $t=11.89 \mathrm{ps}$.

amplitude difference waveform between the defect and nondefect is not uniform, and there are local eigenvalues. The corresponding frequency points of $f=0.25,0.81$, and $1.08 \mathrm{THz}$ at the peak of the amplitude difference are selected for imaging, and the corresponding detection results are illustrated in Figure 6.

3.3. Absorption Coefficient and Imaging. Figure 7 shows the waveform of the absorption coefficient and its amplitude difference between the defect and nondefect in the specimen. Figure 7 (a) clearly shows that the absorption coefficient of the nondefect site remains relatively stable. The overall trend of the absorption coefficient of the defect site increases slowly with increasing frequency, and its value is significantly higher than that of the nondefect site. The increase of absorption coefficient is mainly caused by the structural destruction at the defect of CFRP, which is related to the composite material's complex refractive index to terahertz light. In addition, it is clearly found that the difference of 


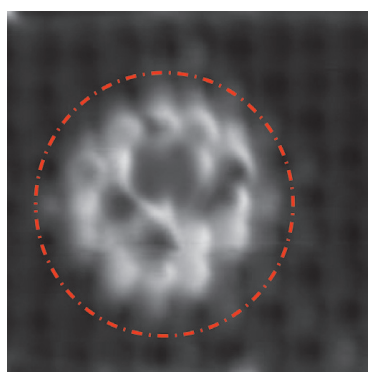

(a)

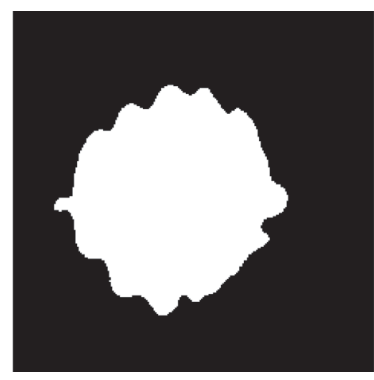

(b)

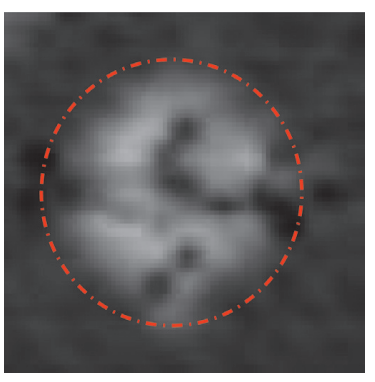

(c)

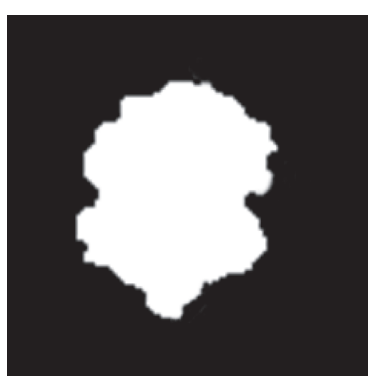

(d)

FIGURE 4: Detection images and extraction results at defect of specimen: (a) time-domain signal imaging; (b) defect extraction result of timedomain signal imaging; (c) infrared imaging; (d) defect extraction result of infrared imaging.

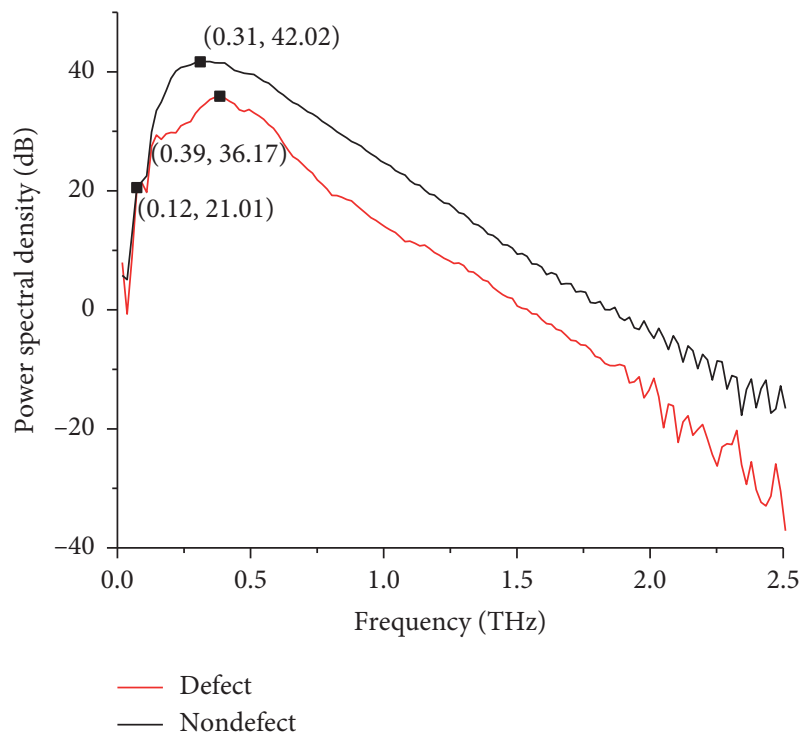

(a)

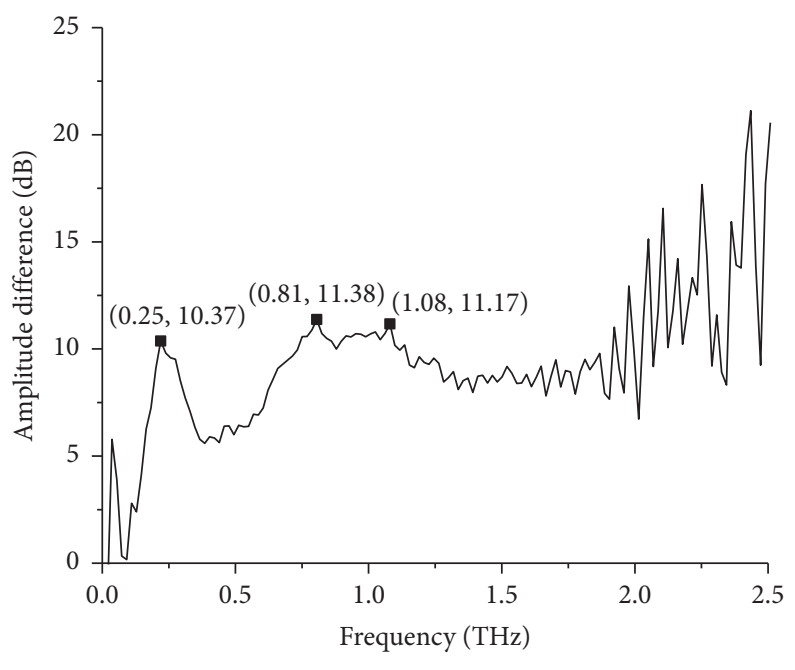

(b)

FIgure 5: (a) Power spectral density waveform. (b) Power spectral density amplitude difference.

absorption coefficient is small when the frequency is less than $0.12 \mathrm{THz}$, and the noise region appears at $2.0 \mathrm{THz}$. Comparing Figure 7(a) with Figure 5(a), the absorption coefficient of the defect is inversely related to its power spectral density. In Figure 7(b), obvious characteristic peaks exist at $0.22,0.81$, and $1.08 \mathrm{THz}$ that correspond to the characteristic points of the power spectral density amplitude difference in Figure 5(b). The aforementioned characteristic peaks are considered to be caused by the fiber structure, epoxy resin, and defects in the specimens [16, 23]. The corresponding frequency points $f=0.22,0.81$, and $1.08 \mathrm{THz}$ corresponding to the peak value difference of the absorption coefficient between the defect and nondefect are selected for imaging, and the corresponding detection results are shown in Figure 8.

Comparing the results of Figures 6 and 8, it is concluded that the image with a larger amplitude difference between 0.81 and $1.08 \mathrm{THz}$ is better than that with a smaller amplitude difference at $f=0.22$ (or $f=0.25 \mathrm{THz}$ ). Moreover, the aforementioned images only show a small number of severely damaged areas in the impact center, and the delamination damage around the specimen is not considered. Therefore, the contrast of the images is relatively small, and the imaging effect is correspondingly low. Comparing the imaging results in the time domain and frequency domains, the imaging results in the time domain are better than those in the frequency domain.

\section{Conclusions}

In this paper, the impact damage defects in CFRP composite laminates were investigated by terahertz time-domain spectroscopy and imaging technology. The maximum peak, minimum peak, maximum flight time, minimum flight time, and characteristic points of differences between peak and amplitude and between power spectral density and absorption coefficient of frequency-domain signals were applied. The time- and frequency-domain measurement data were compared and analyzed, and the imaging analyses of time-domain, power spectral density, and absorption coefficient data were carried out separately according to the spectral characteristics of the defects. Results show that 


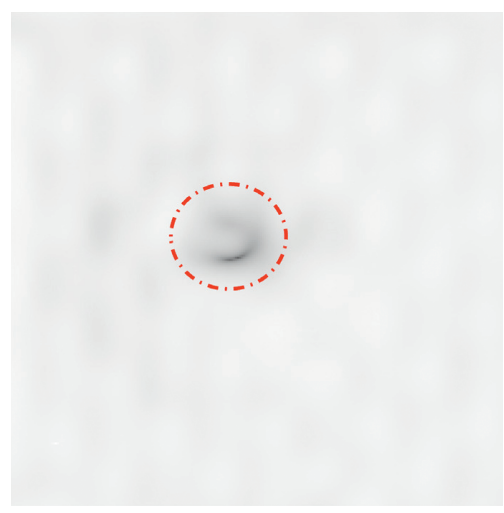

(a)

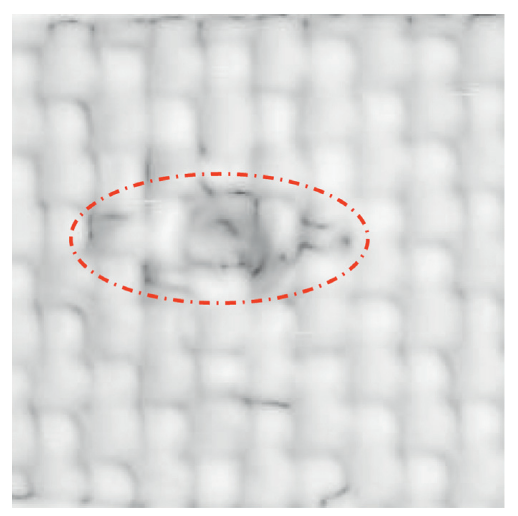

(b)

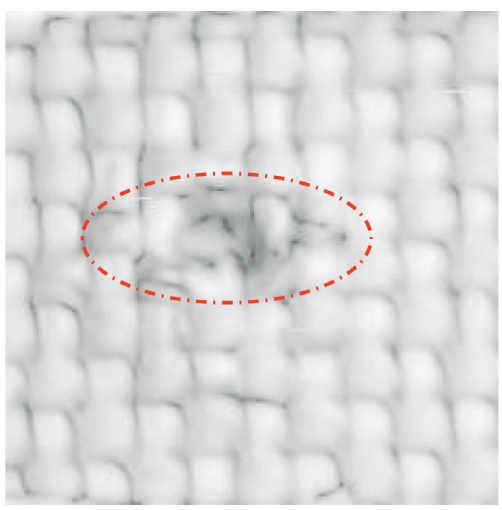

(c)

FIgURE 6: Imaging of power spectral density at defect of specimen: (a) $f=0.25 \mathrm{THz}$; (b) $f=0.81 \mathrm{THz}$; (c) $f=1.08 \mathrm{THz}$.

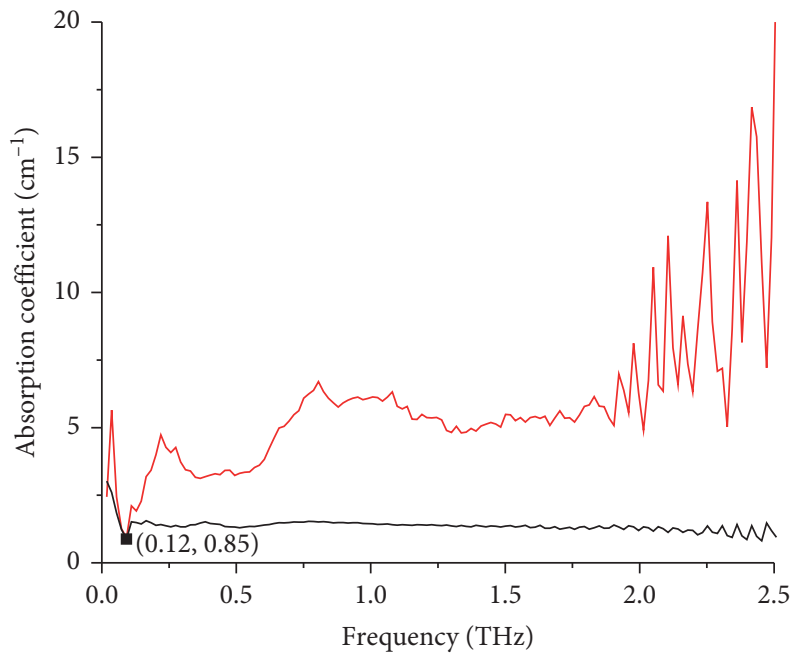

Defect

Nodefect

(a)

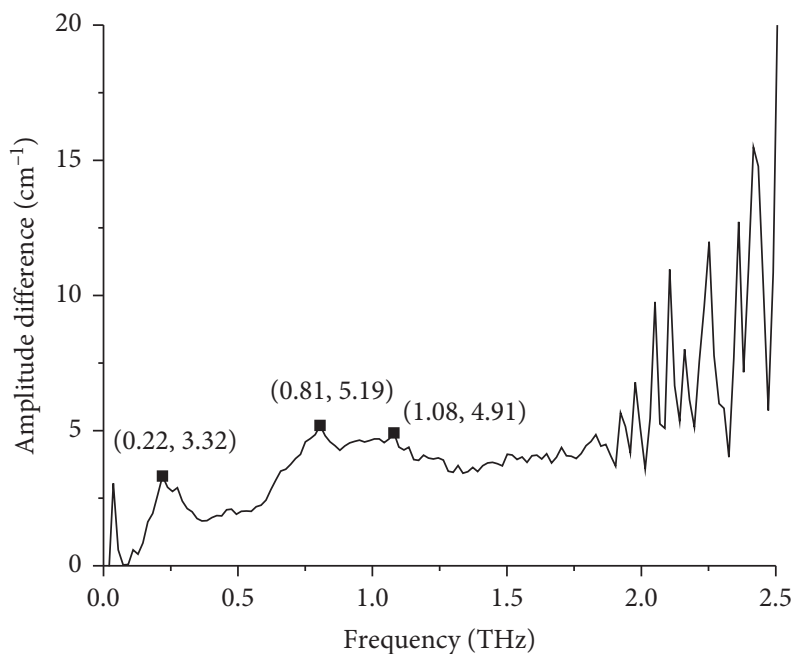

(b)

Figure 7: (a) Absorption coefficient waveform. (b) Absorption coefficient amplitude difference.

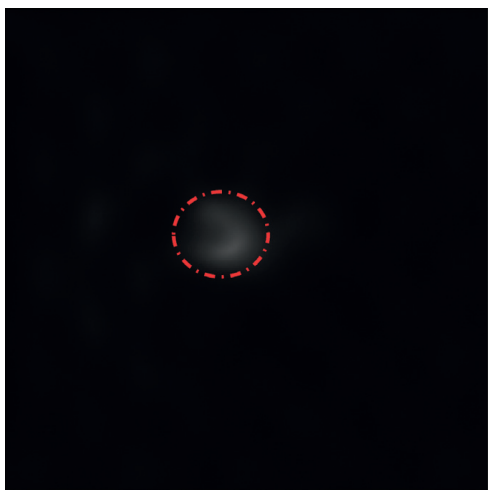

(a)

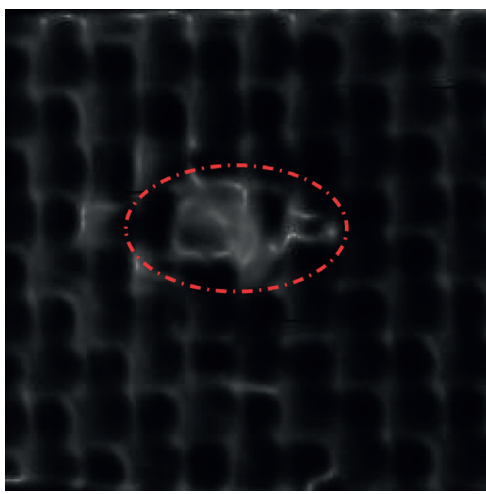

(b)

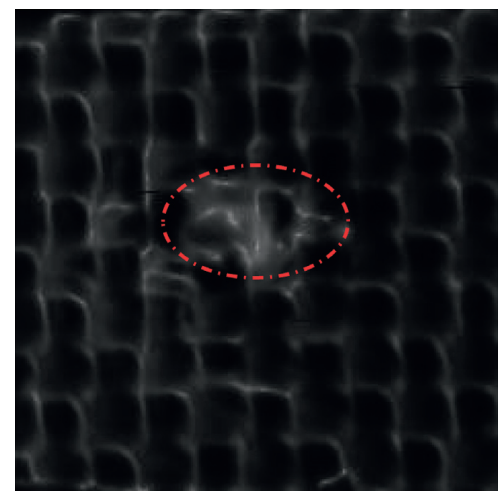

(c)

FIGURE 8: Imaging of absorption coefficient at defect of specimen: (a) $f=0.22 \mathrm{THz}$; (b) $f=0.81 \mathrm{THz}$; (c) $f=1.08 \mathrm{THz}$. 
reflective terahertz imaging technology can be used to effectively distinguish low-velocity impact damage defects in carbon-fiber-reinforced composite laminates in the range of 0.12-2.0 THz band; furthermore, the time-domain signals of the defect area have an attenuation and delay comparable with those of the nondefect area. In the frequency domain, the power spectral density of defects is lower than that of nondefects. The power spectral densities of defects and nondefects decrease with increasing frequency after reaching peaks at 0.31 and $0.39 \mathrm{THz}$, respectively. In addition, the absorption coefficient at the nondefect sites remains stable, and the absorption coefficient at the defect sites increases slowly with fluctuation, which is generally higher than that of the nondefect sites. By analyzing terahertz time- and frequency-domain signals and amplitude differences and performing imaging analysis according to its local eigenvalues, the qualitative detection of low-velocity impact damage defect in experimental specimens can be measured. However, the imaging results are quite consistent from various imaging modes. Comparing the time- and frequency-domain imaging results, the imaging results of the time-domain detection are obviously better than those of frequency-domain detection. Thus, terahertz time-domain spectroscopy and imaging technology has great potential in the quantitative detection of low-velocity impact defects, and it is worthy of further study.

\section{Data Availability}

The data used to support the findings of this study are available from the corresponding author upon request.

\section{Conflicts of Interest}

The authors declare that there are no conflicts of interest regarding the publication of this article.

\section{Acknowledgments}

This work was financially supported by the National Key R\&D Program of China (nos. 2018YFC0809500 and 2017YFF0106303), Key Project of Sichuan Department of Science and Technology (nos. 2018GZ0497 and 2019YFG003), and Project of Civil Aviation Flight University of China (nos. BJ2016-04, J2018-56, and CJ2019-01). The authors also gratefully acknowledge the help of Li Peng.

\section{References}

[1] K. B. Katnam, L. F. M. Da Silva, and T. M. Young, "Bonded repair of composite aircraft structures: a review of scientific challenges and opportunities," Progress in Aerospace Sciences, vol. 61, pp. 26-42, 2013.

[2] N. Keršienè, L. Raslavičius, A. Keršys, R. Kažys, and E. Žukaus, "Energo-mechanical evaluation of damage growth and fracture initiation in aviation composite structures," Polymer-Plastics Technology and Engineering, vol. 55, no. 11, pp. 1137-1144, 2016.

[3] A. Baker, A. J. Gunnion, and J. Wang, "On the certification of bonded repairs to primary composite aircraft components," The Journal of Adhesion, vol. 91, no. 1-2, pp. 4-38, 2015.

[4] S. Sfarra, C. Ibarra-Castanedo, C. Santulli et al., "Falling weight impacted glass and basalt fibre woven composites inspected using non-destructive techniques," Composites Part B: Engineering, vol. 45, no. 1, pp. 601-608, 2013.

[5] Y. Li, W. Zhang, Z.-W. Yang, J.-Y. Zhang, and S.-J. Tao, "Lowvelocity impact damage characterization of carbon fiber reinforced polymer (CFRP) using infrared thermography," Infrared Physics \& Technology, vol. 76, pp. 91-102, 2016.

[6] A. Katunin, K. Dragan, and M. Dziendzikowski, "Damage identification in aircraft composite structures: a case study using various non-destructive testing techniques," Composite Structures, vol. 127, pp. 1-9, 2015.

[7] I. Taraghi and A. Fereidoon, "Non-destructive evaluation of damage modes in nanocomposite foam-core sandwich panel subjected to low-velocity impact," Composites Part B: Engineering, vol. 103, pp. 51-59, 2016.

[8] R. Quintero, F. Simonetti, P. Howard, J. Friedl, and A. Sellinger, "Noncontact laser ultrasonic inspection of ceramic matrix composites (CMCs)," NDT \& E International, vol. 88, pp. 8-16, 2017.

[9] I. Pelivanov, T. Buma, J. Xia, C.-W. Wei, and M. O’Donnell, "A new fiber-optic non-contact compact laser-ultrasound scanner for fast non-destructive testing and evaluation of aircraft composites," Journal of Applied Physics, vol. 115, no. 11, Article ID 113105, 2014.

[10] V. P. Vavilov, "Dynamic thermal tomography: recent improvements and applications," NDT \& E International, vol. 71, pp. 23-32, 2015.

[11] U. Polimeno, D. P. Almond, B. Weekes, and E. W. J. Chen, “A compact thermosonic inspection system for the inspection of composites," Composites Part B: Engineering, vol. 59, pp. 67-73, 2014.

[12] A. Poudel, K. R. Mitchell, T. P. Chu, S. Neidigk, and C. Jacques, "Non-destructive evaluation of composite repairs by using infrared thermography," Journal of Composite Materials, vol. 50, pp. 1-13, 2015.

[13] D. Findeis, J. Gryzagoridis, and C. Lombe, "Comparing infrared thermography and ESPI for NDE of aircraft composites," Insight-Non-Destructive Testing and Condition Monitoring, vol. 52, no. 5, pp. 244-247, 2010.

[14] A. M. Amaro, P. N. B. Reis, M. F. S. F. De Moura, and J. B. Santos, "Damage detection on laminated composite materials using several NDT techniques," Insight-Non-Destructive Testing and Condition Monitoring, vol. 54, no. 1, pp. 14-20, 2012.

[15] M. E. Ibrahim, "Nondestructive evaluation of thick-section composites and sandwich structures: a review," Composites Part A: Applied Science and Manufacturing, vol. 64, pp. 36-48, 2014.

[16] J. Zhang, C. C. Shi, Y. T. Ma et al., "Spectroscopic study of terahertz reflection and transmission properties of carbonfiber-reinforced plastic composites," Optical Engineering, vol. 54, no. 5, Article ID 054106, 2015.

[17] D. K. Hsu, K. S. Lee, J. W. Park et al., "NDE inspection of terahertz waves in wind turbine composites," International Journal of Precision Engineering \& Manufacturing, vol. 13, no. 5, pp. 1183-1189, 2012.

[18] C. Jördens, M. Scheller, S. Wietzke et al., "Terahertz spectroscopy to study the orientation of glass fibres in reinforced plastics," Composites Science and Technology, vol. 70, no. 3, pp. $472-477,2010$. 
[19] A. Abina, U. Puc, A. Jeglič, and A. Zidanšek, "Structural characterization of thermal building insulation materials using terahertz spectroscopy and terahertz pulsed imaging," NDT \& E International, vol. 77, pp. 11-18, 2016.

[20] L. Y. Xing, H. L. Cui, C. C. Shi et al., "Experimental study of PMI foam composite properties in terahertz," Spectroscopy and Spectral Analysis, vol. 35, no. 12, pp. 3319-3324, 2015.

[21] Q. Wang, X. Y. Li, T. Y. Chang et al., "Terahertz time-domain spectroscopic study of aircraft composite and matrix resins," Spectroscopy and Spectral Analysis, vol. 38, no. 9, pp. 27062712, 2018.

[22] N. Palka, R. Panowicz, and F. Ospald, "3D non-destructive imaging of punctures in polyethylene composite armor by THz time domain spectroscopy," Journal of Infrared, Millimeter, and Terahertz Waves, vol. 36, no. 9, pp. 770-788, 2015.

[23] J. Zhang, W. Li, H.-L. Cui et al., "Nondestructive evaluation of carbon fiber reinforced polymer composites using reflective terahertz imaging," Sensors, vol. 16, no. 6, pp. 875-886, 2016. 